\title{
Clasificación y tratamiento de náusea y vómito postoperatorio en el paciente oncológico sometido a cirugía
}

\author{
Postoperative nausea and vomiting in oncologyc patient: \\ Clasification and treatment
}

Giancarlo Ferretiz López¹, Luis Felipe Cuéllar Guzmán, Neftali Cárdenas Herrera

\begin{abstract}
Recognizing the importance of preventing and treating postoperative nausea and vomiting (PONV) in a timely manner is essential since it is considered to be one of the most frequent problems in surgical patients within the postoperative recovery unit, since it represents an incidence of $30 \%$ to $50 \%$, significantly prolonging the length of stay in the postoperative recovery unit. It is important to familiarize ourselves with the predictors of postoperative nausea and vomiting (PONV) and new concepts such as post-discharge nausea and vomiting (PDNV), nausea and vomiting induced by chemotherapy. (QINAV) and radiation induced nausea and vomiting (RINV). So the anesthesiologist in this situation must determine the necessary strategies according to the current characteristics of the patient, disease, evolution (stage) and previous treatment for its classification of emetic risk and according to the new strategies described in the literature, propose the resources and the ideal treatment which will favor to reduce and prevent this complication and favor an opportune discharge. So we present extensive review of this topic.
\end{abstract}

\section{RESUMEN}

El reconocer la importancia de prevenir y tratar de forma oportuna las náuseas y vómito postoperatorio (NVPO) es fundamental, ya que se considera que es uno de los problemas más frecuentes en los pacientes quirúrgicos dentro de la Unidad de Recuperación Postoperatoria, ya que representa una incidencia del $30 \%$ al 50\%, llegando a prolongar significativamente el tiempo de estadía

\section{Key words:}

Horner syndrome, neuraxial anesthesia, obstetric anesthesia

Palabras clave:

Síndrome de Horner, anestesia neuroaxial, anestesia obstétrica

Instituto Nacional de Cancerología, México.

Fecha de recepción: 20 de abril de 2019

Fecha de aceptación: 25 de abril de 2019

Orcid

https://orcid.org/0000-0001-6860-8774

Correspondencia:

Dr. Giancarlo Ferretiz López

Email: dcferretiz@hotmail.com 
en la Unidad de Recuperación Postoperatoria. Es importante familiarizarnos con los factores predictivos de la náusea y vómito postoperatorio (NVPO) y los nuevos conceptos como las náuseas y vómito postalta (NVPA), náusea y vómitos inducidos por quimioterapia. (NAVIQ) y náusea y vómito inducidas por radioterapia (NVIR), por lo que el anestesiólogo ante esta situación debe determinar las estrategias necesarias de acuerdo a las características actuales del paciente, enfermedad, evolución (estadio) y tratamiento previo para su clasificación de riesgo emético, y de acuerdo a las nuevas estrategias descritas en la literatura, proponer los recursos y el tratamiento ideal lo que favorecerá a reducir y prevenir esta complicación y favorecer a un egreso oportuno. Por lo que a continuación presentamos una amplia revisión del tema.

\section{Introducción}

a anestesiología adquirió un rol fundamental en torno a los pacientes con cáncer, no solo se debe considerar el manejo perioperatorio por el anestesiólogo ya que puede disminuir el estrés quirúrgico y metabólico (respuestas neuroendocrinas, inflamatorio e inmunosupresión), siendo necesario implementar protocolos específicos en náusea y vómito postoperatorio ya que es la complicación mayormente asociada a una estancia hospitalaria prolongada o reingreso hospitalario.

Los posibles factores de riesgo para la presencia de náusea y vómito postoperatorio incluyen los procedimientos intraabdominales, laparoscópicos, ortopédicos, ginecológicos, otorrinolaringológicos, tiroides, cirugía plástica, de mama y procedimientos neuroquirúrgicos. La náusea y vómito postoperatorio (NVPO) es un problema frecuente en los pacientes quirúrgicos, con una incidencia de $50 \%$ y de $30 \%$ respectivamente. En pacientes con alto riesgo de presentar esta complicación pueden aumentar la incidencia de hasta el $80 \%$. La NVPO puede prolongar significativamente el tiempo de estadía en la Unidad de Recuperación Postoperatoria (URP) con incremento de forma relevante en los costos de la cirugía. Cada episodio de vómitos puede llegar a aumenta en 20 min la estadía en una Unidad de Recuperación Postoperatoria.

La náusea es definida como la sensación desagradable e inminente de vomitar, puede ocurrir sola o acompañada de vómitos, definidos como la eyección enérgica del contenido gastrointestinal a través de la boca mediante contracciones involuntarias de la musculatura de la pared torácica y abdominal. Estos síntomas se deben diferenciar de las arcadas, que consisten en movimientos espasmódicos de la musculatura toraco-abdominal en la que coexiste una inspiración forzada contra un cierre de la boca y de la glotis, sin llegar a exteriorizar el contenido gástrico.

La náusea o el vómito están relacionados con múltiples causas como las alteraciones a nivel del tracto gastrointestinal (reflujo gastroesofágico, alteraciones funcionales, gastroparesia, obstrucción mecánica, etc.), infecciones víricas o bacterianas, alteraciones endocrinas (embarazo, hipotiroidismo), alteraciones a nivel del sistema nervioso central (aumento de la presión intracraneal, convulsiones, migraña, etc.). En el campo de la oncología pueden estar relacionados con los tratamientos de radioterapia y/o quimioterapia o con el propio cáncer.

Claudio Nazar en su artículo de revisión refiere que la náuseas y vómitos postoperatorios (NVPO) son un problema frecuente en los pacientes quirúrgicos, con una incidencia de $50 \%$ y de $30 \%$ respectivamente. En pacientes con alto riesgo de presentar esta complicación pueden llegar a una incidencia de hasta el $80 \%$. Las (NVPO) pueden prolongar significativamente el tiempo de estadía en la Unidad de Recuperación Postoperatoria (URP) y aumentar de forma relevante los costos de la cirugía. Cada episodio de vómitos aumenta en 20 min la estadía en una Unidad de Recuperación Postoperatoria URP[1].

Las (NVPO) son la principal causa de hospitalización no programada en cirugía ambulatoria, en el cual el objetivo debe ser la profilaxis de las NVPO más que su tratamiento, con el fin de disminuir significativamente su incidencia, molestias secundarias del paciente y las varias complicaciones asociadas, como alteraciones hidroelectrolíticas, dehiscencia de suturas, aspiración pulmonar de contenido gástrico, aumento de la presión intracraneana, entre otras[1].

Algunas de las complicaciones propias de la (NVPO) son la insatisfacción por la anestesia, la neumonitis por aspiración, la dehiscencia de suturas, la ruptura esofágica, el enfisema subcutáneo, el neumotórax bilateral, los hematomas y el retardo del alta 
hospitalaria[2].

La clasificación actual de náuseas y vómitos, náusea y vómito postoperatorio (NVPO), náusea y vómito postalta (NVPA), náusea y vómitos inducidos por quimioterapia (NAVIQ) y náusea y vómitos inducidos por radioterapia (NAVIR).

\section{Factores de riesgo para náusea y vómitos postoperatorios}

Apfel et al, realizaron en 2012 una revisión sistemática de 22 estudios prospectivos, con más de 500 pacientes cada uno, para identificar factores predictores independientes de (NVPO). Los resultados demostraron que, de los factores de riesgo relacionados con el paciente, el sexo femenino fue el factor predictor más importante, seguido de historia de (NVPO) en cirugías previas y/o cinetosis, y no fumar. A mayor edad, disminuye significativamente el riesgo de (NVPO), especialmente sobre los 50 años. De los factores de riesgo relacionados con la cirugía-anestesia, el uso de anestésicos inhalatorios fue el predictor más importante, seguido de duración de la anestesia, utilización de opioides postoperatorios y uso de óxido nitroso. La evidencia encontrada para el tipo de cirugía como factor de riesgo independiente de (NVPO) resultó controversial.

Sin embargo, sí se identificaron 3 tipos de cirugías como factores predictores importantes para (NVPO): colecistectomía, cirugía laparoscópica y cirugía ginecológica. En cirugías ambulatorias en EE.UU., la incidencia de náusea y vómito posteriores al alta de una cirugía ambulatoria (NVPO en CA), entre el día del alta y el segundo día postoperatorio, fue de 37\%[3].

El análisis identificó 5 predictores independientes estadísticamente significativos para (NVPO en CA): náuseas en Unidad de Recuperación Postoperatoria, edad menor de 50 años, administración de opioides postoperatorios, género femenino e historia de NVPO en anestesias previas, en la que un puntaje de 0 puntos representa el $7 \%$ a presentar NVPO, 1 punto $20 \%, 2$ puntos $28 \%$ (Riesgo Bajo), 3 puntos $53 \%, 4$ puntos 60\% (Riesgo Medio), 5 puntos $89 \%$ (Riesgo Alto)[17]. Ninguno de los factores mencionados por sí solo es suficiente para evaluar el riesgo de NVPO o para tomar decisiones sobre qué antieméticos profilácticos utilizar. El uso de escalas de riesgo y las respectivas profilaxis para NVPO han demostrado disminuir significativamente la tasa de esta complicación. Aunque son una aproximación objetiva para evaluar el riesgo de un paciente de presentar NVPO, ya sea en pacientes hospitalizados o cirugía ambulatoria, tienen una sensibilidad y especificidad entre $65 \%$ y $70 \%[3]$.
Las estrategias generales que se pueden utilizar para reducir el riesgo basal de (NVPO) son evitar la anestesia general, prefiriendo la anestesia regional (evidencia tipo 1(A), utilizar propofol intravenoso (IV) para la inducción y mantención de la anestesia general (1A), evitar el uso de óxido nitroso (1A), evitar el uso de anestésicos inhalatorios (2A), minimizar el uso intraoperatorio y postoperatorio de opioides (1A), hidratación iv abundante (1A)[4].

\section{Náusea y vómito postalta (NVPA)}

Los estudios de Apfel et al, identificaron que los factores de riesgo de náuseas y vomito postalta NVPA son diferentes en las primeras $48 \mathrm{~h}$ que a partir del día 3 al 7, cuyos factores se pueden clasificar de forma independientes para NVPA incluyendo, sexo (femenino), edad menor de 50 años, historia de náusea y vómito postoperatorio (NVPO), el uso de opioides en Unidad de Recuperación Postanestésica (URPA) y la presencia de náuseas. Se describe que el dolor es el factor de riesgo más importante asociado a la aparición de NVPA entre el $3^{\text {er }}$ y $7^{\text {to }}$ día tras la cirugía mayor ambulatoria. Es importante destacar que ni el tipo de cirugía ni el estado no fumador son predictores independientes de náusea y vómito postalta (NVPA). Los pacientes que tienen náuseas en la Unidad de Recuperación Postalta (URPA) tienen un riesgo 3 veces mayor de NVPA[5].

Cabe destacar también que el uso de ondansetrón en el intraoperatorio disminuye la incidencia de (NVPO) en la (URPA), pero no tras el alta, y lo contrario ocurre con el uso de corticoides, que no parecen tener un efecto protector en la (URPA), pero sí ante las NVPA. La anestesia total intravenosa solo tiene un efecto antiemético durante las primeras horas del postoperatorio y, por tanto, en CMA, su utilización no debería sustituir a la administración de un antiemético.

\section{Náusea y vómitos inducidos por quimioterapia (NAVIQ)}

Se presenta hasta en el $80 \%$ de los pacientes y puede tener un impacto importante en la calidad de vida de los pacientes. Por ello, el objetivo principal del oncólogo médico que asiste a un paciente es evitar la aparición de náuseas y/o vómitos. Su etiología, y fisiopatología ocurre por diversos mecanismos.

Se reconoce un mecanismo central, en donde en la zona gatillo quimiorreceptora localizada en el área postrema (AP) del suelo del cuarto ventrículo recibe estímulos eméticos a través de la sangre y el 
líquido cefalorraquídeo rotoninérgicos, histamínicos y de neurocinina- 1 . Una vez activada la zona gatillo se liberan neurotransmisores que estimulan el centro del vómito, el cual no tiene localización anatómica específica, aunque se considera como un "área» de receptores situada en el núcleo solitario; el centro del vómito recibe fibras aferentes de la zona gatillo quimiorreceptora, el tracto espinovestibular, la corteza cerebral y estímulos del sistema digestivo a través del nervio vago y los nervios esplácnicos[6].

El mecanismo periférico. El efecto de la quimioterapia puede producir la liberación de diversos neurotransmisores (serotonina, colecistoquinina y neurocinina- 1) a través de la vía de las células enterocromafines. Estos neurotransmisores estimulan las vías vagales aferentes que transmiten el impulso nervioso al tracto solitario y posteriormente al centro del vómito[6]. Por último, se tiene considerada que la estimulación directa del área postrema por fármacos citotóxicos, también ha sido propuesta como un mecanismo adicional de emesis inducida por quimioterapia[7].

Las náuseas y vómitos inducidos por quimioterapia (NAVIQ) pueden ser clasificados en diferentes tipos como: Agudos: La cual se presentan en el transcurso de las primeras 24 horas desde la administración de la quimioterapia. La intensidad de la emesis aguda presenta su pico máximo a las 5-6 horas posteriores a la administración de la quimioterapia y entre los factores predisponentes se observa que es más frecuente en gente joven (menores de 50 años) y en mujeres con bajo consumo de alcohol. Diferidos o tardíos: Se manifiestan más de 24 horas después de la administración de quimioterapia, la que se encuentra mayormente relacionada con (cisplatino, ciclofosfamida) así como otros fármacos como lo son (doxorrubicina e ifosfamida). La anticipatoria: Se presentan antes del comienzo de un nuevo ciclo de quimioterapia como respuesta a estímulos condicionados como olores, objetos o sonidos de la sala de tratamiento. Constituyen una respuesta condicionada clásica que generalmente se presenta después de tres o cuatro tratamientos previos de quimioterapia, tras los cuales la persona experimentó náuseas y vómitos agudos 0 diferidos[8].

La presentación episódicos: Son los vómitos que se presentan a pesar del tratamiento profiláctico con antieméticos y para los que se necesita tratamiento adicional. $Y$, por último, las de tipo refractarios son aquellas náuseas y vómitos que no responden al tratamiento.

En la población (oncológico) existen múltiples factores que están relacionados con las náuseas y vómi- tos inducidos por la quimioterapia, los cuales se pueden dividir en: Factores asociados al tratamiento considerado como el factor más importante el potencial emético del antineoplásico, en donde en diversos estudios como Grunberg et al., desarrollaron en el año 2011 una clasificación y un algoritmo de la capacidad emetizante aguda de los agentes quimioterapéuticos y esquemas de quimioterapia combinada[8].

\section{Los factores relacionados con el paciente}

En los cuales los episodios previos de vómitos con la quimioterapia aumentan el riesgo de NAVIQ, el sexo femenino presenta un mayor riesgo en comparación con los hombres, los pacientes más jóvenes son más susceptibles a NAVIQ que los pacientes de edad avanzada, los pacientes con antecedentes de consumo de alcohol son menos susceptibles a NAVIQ, así mismo se menciona a los pacientes metabolizadores rápidos de ciertos antagonistas de los receptores de 5HT3, son más susceptibles a NAVIQ severos, ciertos polimorfismos en los receptores de 5HT3 pueden conferir también un mayor riesgo de NAVIQ y, por último, la emesis anticipatoria ocurre en pacientes con pobre control de la emesis aguda o tardía con quimioterapia previa[22],[23], siendo actualizada por Roila F et al., en el año 2016 en la que se hizo una división de los agentes antineoplásicos en 4 niveles, según el porcentaje en que los pacientes experimentan vómitos agudos cuando no reciben la profilaxis antiemética[9].

\section{Clasificación de acuerdo al potencial emético de los antineoplásicos}

En las actualizaciones presentadas por Roila $F$, Molassiotis A, Paul J. Hesketh de las guías médicas de NVPO la Multinational Association of Supportive Care in Cancer/European Society for Medical Oncology (MASCC/ESMO), la Sociedad Americana de Oncología Clínica (ASCO) y la National Comprehensive Cancer Network (NCCN) (Tabla 1)[7],[9].

\section{Las náuseas anticipatorias}

Con un alto componente emocional, la profilaxis de este tipo de eventos pasa por una buena prevención en los primeros ciclos y el uso de benzodiacepinas en casos de difícil control. Deben usarse las dosis y la vía de administración recomendada para cada antagonista de 5HT3. Si como antagonista de NK1R elegimos aprepitant, este debe administrarse en dosis 


\section{Tabla 1}

\begin{tabular}{ll}
\hline Riesgo alto (90\%) & $\begin{array}{l}\text { Adriamicina }\left(+60 \mathrm{mg} / \mathrm{m}^{2}\right) \text {, carmustina, cisplatino, dacarbacina, estreptozotocina, } \\
\text { mecloretamina, epirrubicina }\left(90 \mathrm{mg} / \mathrm{m}^{2}\right), \text { ifosfamida }\left(+10 \mathrm{~g} / \mathrm{m}^{2}\right) \text {. (Combinación de } \\
\text { antraciclina/ciclofosfamida. Carmustina, cisplatino, ciclofosfamida) y con administra- } \\
\text { ción vía oral (hexametilmelamina, procarbazina) }\end{array}$ \\
Riesgo moderado (30\%-90\%) & $\begin{array}{l}\text { Alemtuzumab, azacitidina, bendamustina, carboplatino, daunorubicina, doxorubicina, } \\
\text { idarubicina, ifosfamida, irinotecan, oxaliplatino). Administración vía oral. (Bosutinib, } \\
\text { ceritinib, crizotinib, ciclofosfamida, imatinib. }\end{array}$ \\
Riesgo bajo (10\%-30\%) & $\begin{array}{l}\text { Docetaxel, eribulin, etoposido, 5Fu, gemcitabina, ixabepilone, metotrexate, mitomici- } \\
\text { na, mitoxantrone, nabpaclitaxel, paclitaxel, doxorrubicina, pertuzumab, temsirolimus, } \\
\text { topotecan). Administración vía oral (capecitabina, etoposido, lapatinib, lenalidomide, } \\
\text { olaparib, nilotinib, pazopanib, ponatinib, regorafenib, sunitinib }\end{array}$ \\
Riesgo mínimo (menor de 10\%) & $\begin{array}{l}\text { Bevacizumab, bleomicina, busulfan, cladribina, fludarabina, pralatrexate, rituxim, vin- } \\
\text { blastine, vincristine, vinorelbine). Y en administración vía oral (clorambucilo, erlotinib, } \\
\text { gefitinib, hydroxyurea, melfalan, metotrexate, pomalidomide, ruxolitinib, sorafenib, } \\
\text { 6-thioguanine, vemurafenib, vismodegib }\end{array}$ \\
\hline
\end{tabular}

de 125 mg vía oral en dosis única una hora antes de la administración de la quimioterapia y repetirse con 80 mg los días 2 y 3. Si usamos fosaprepitant bastará con una dosis única de $150 \mathrm{mg}$ intravenosos antes de la quimioterapia. Si usamos fosaprepitant bastará con una dosis única de $150 \mathrm{mg}$ intravenosos antes de la quimioterapia, no se requiere en este caso profilaxis para la emesis retardada. Si como antagonista de NK1R elegimos aprepitant, éste debe administrarse en dosis de 125 mg vía oral en dosis única una hora antes de la administración de la quimioterapia y repetirse con 80 mg los días 2 y 3[9].

\section{Náuseas y vómitos refractarios}

En ocasiones, a pesar de usar los fármacos y las dosis adecuadas como profilaxis, el paciente puede experimentar náuseas o vómitos persistentes, siendo necesario un diagnóstico diferencial detallado para descartar causas intercurrentes como pueden ser: fármacos opioides, antibióticos, obstrucción intestinal, hipercalcemia o hipertensión intracraneal, entre otros[9]. En estos casos estaría indicado aumentar el escalón terapéutico o añadir fármacos coadyuvantes como benzodiacepinas, antagonistas dopaminérgicos (haloperidol, olanzapina) o metoclopramida en dosis altas.

\section{Náuseas y vómitos en enfermedad avanzada}

En el paciente con enfermedad avanzada, las náuseas y los vómitos pueden deberse a múltiples complicaciones: como lo son el aumento de presión intracraneal (metástasis cerebrales, carcinomatosis), alteracio- nes hidroelectrolíticas (hipercalcemia, hiponatremia), obstrucción mecánica (vaciamiento gástrico, ascitis, hepatomegalia, obstrucción intestinal, alteraciones neurológicas (disfunción vestibular, neuropatía paraneoplásica), farmacológica (opioides). El tratamiento de elección en pacientes con enfermedad avanzada y mal estado general se basa en metoclopramida, antagonistas dopaminérgicos (haloperidol, levomepromazina, olanzapina), anticolinérgicos y antisecretores del tipo análogos de somatostatina[9].

\section{Náusea y vómito inducidos por radioterapia (NVIR)}

Aunque el problema de las náuseas y vómitos no es tan grave como en el escenario de algunos fármacos antineoplásicos, sí puede serlo en los servicios de radioterapia. En consecuencia, es necesario determinar cuáles son las situaciones en las que es imperativo iniciar el manejo profiláctico para náuseas y vómitos inducidos por radioterapia (NVIR). Estos síntomas, si no son tratados, pueden deteriorar la calidad de vida, complicar otros síntomas y, lo más importante, hacer que se suspenda temporalmente el manejo con radioterapia (RT). La sintomatología de la (NVIR) depende básicamente del sitio anatómico del tratamiento con RT, el volumen de órganos críticos (incluidos esófago, estómago e intestino delgado) y la concomitancia con algunos fármacos antineoplásicos utilizados como radiosensibilizadores[10],[11].

Los casos de radiación corporal total, el riesgo es muy alto y casi en todos los casos se va a presentar, en este estado clínico, las causas de las náuseas y vómitos inducidos por radioterapia (NVIR), no solo 
es por la liberación de serotonina por las células enterocromafines intestinales, sino también de una gran necrosis tisular, cuyas células liberan endotoxinas, así como la hipertensión intracraneal con irritación de la corteza cerebral. Al final, por diferentes caminos, hay una estimulación del centro de vómito y se produce la emesis[10].

Los casos de radioterapia corporal total (previo al trasplante de médula ósea), el paciente, además, recibirá quimioterapia, por lo que el riesgo no sólo será mayor, sino que el período de la sintomatología será más prolongado. Lo anterior debe tomarse en cuenta porque los síntomas determinarán el tiempo de administración de los antieméticos. Al utilizar medicamentos como radiosensibilizador es su caso el platino. Las náuseas y vómitos se pueden presentar con cualquier dosis (pero mucho más con las mayores de $50 \mathrm{mg} / \mathrm{m}^{2}$ sc), por lo que es deseable la profilaxis. No obstante, pese a que hay otros fármacos como gemcitabina, vinorelbina, 5-fluoruracilo o capecitabina, que tienen menor potencial emetogénico per se, se deberán evaluar en el escenario de la concomitancia y tomar muy en cuenta el sitio anatómico irradiado[10].

La dosis por fracción también deberá tomarse en cuenta, ya que las de 3 Gy o mayores tienen mayor riesgo de provocar náuseas y vómitos inducidos por radioterapia (NVIR), pero depende del sito anatómico. Por ejemplo, irradiación con 3 o 4 Gy por fracción de lesiones en extremidades por metástasis óseas, nunca se presentará náuseas y vómitos inducidos por radioterapia (NVIR), pero en los campos grandes de tratamiento (superiores a $400 \mathrm{~cm}^{2}$ ), el riesgo es alto[10],[11].

Factores como edad, sexo, comorbilidades, consumo de opiáceos, estado psicológico, etcétera, también deberán ser considerados. Finalmente, es importante señalar que, del grupo de pacientes que reciben $\mathrm{RT}$ y que tienen riesgo bajo de náuseas y vómitos inducidos por radioterapia (NVIR), deberá excluirse del tratamiento con antieméticos a aquellos que reciben $\mathrm{RT}$ sólo en la cabeza. El fármaco antiemético debe administrarse antes de la RT y continuarlo los días o semanas que dure ésta. Puede iniciarse una reducción gradual en la última parte del tratamiento. En casos de administración por varios días, es recomendable agregar un inhibidor de la bomba de protones. En los pacientes en quienes se considera un riesgo bajo, debe preferirse el manejo sólo de rescate[11].

Los pacientes para presentar náuseas y vómitos inducidos por radioterapia (NVIR) se dividen en: Riesgo alto: > 90\% pacientes sometidos radioterapia corporal total; hemicuerpo superior e inferior; Ries- go moderado: 60\%-90\% pacientes con radioterapia en abdomen superior; craneoespinal esófago. Riesgo bajo: 30\%-60\% pacientes sometidos a radioterapia en pelvis, tórax, cuello y cara, cráneo. Riesgo mínimo: $<30 \%$ pacientes con radioterapia en extremidades y mama[24],[25]. El tratamiento antiemético debe administrarse antes de la RT y continuarlo los días o semanas que dure ésta. Puede iniciarse una reducción gradual en la última parte del tratamiento. En casos de administración por varios días, es recomendable agregar un inhibidor de la bomba de protones, en quienes se considera un riesgo bajo, debe preferirse el manejo sólo de rescate. En esta situación siempre deberán excluirse algunos otros factores que estén provocando náuseas o vómitos.

En consecuencia, es necesario cambiar el paradigma acerca de cómo actuar y determinar cuáles son las situaciones en las que es imperativo iniciar el manejo profiláctico para náuseas y vómitos inducidos por radioterapia (NVIR). Los síntomas, si no son tratados, pueden deteriorar la calidad de vida, complicar otros síntomas y, lo más importante, hacer que se suspenda temporalmente el manejo con radioterapia lo que se traduciría en una falta de control tumoral final[10],[11].

Con base en lo anterior las recomendaciones terapéuticas basadas en la SEOM, y las guías internacionales la Multinational Association of Supportive Care in Cancer/European Society for Medical Oncology (MASCC/ESMO), la Sociedad Americana de Oncología Clínica (ASCO) y la National Comprehensive Cancer Network (NCCN).

Los pacientes altamente eméticos la terapéutica se divide en dos la cual comprende la (fase aguda) o Día 1: en donde los medicamentos de elección comprenden al antagonista del receptor (5HT3) más antagonista (NK1R) más dexametasona $12 \mathrm{mg} \pm$ olanzapina $10 \mathrm{mg}$. (fase tardía día 2 - 40): dexametasona $8 \mathrm{mg}$ \pm olanzapina 10 mg/día. En el caso de esquemas con (antraciclinas-ciclofosfamida), empleado en el cáncer de mama, no existe evidencia sobre el uso de dexametasona los días 2-4 si previamente hemos usado antagonista NK1R. Pacientes moderadamente eméticos (fase aguda): antagonista 5HT3* más dexametasona $12 \mathrm{mg}$; (fase tardía): dexametasona $8 \mathrm{mg} \pm$ antagonistas 5HT3. Moderadamente eméticos basados en carboplatino (fase aguda): NK1R antagonista, más antagonista 5HT3, más dexametasona 12 mg; (fase tardía): no necesitan. Moderadamente eméticos (sin carboplatino) (fase aguda): antagonista 5HT3, más dexametasona $12 \mathrm{mg}$; (fase tardía): dexametasona 8 $\mathrm{mg} \pm$ antagonistas 5HT3[12].

Varios estudios, entre ellos una fase III, han de- 
mostrado que el uso concomitante de NK1R antagonista aumenta el control de las (NAVIQ) hasta un $15 \%$. Por ello, el uso de NK1R antagonistas en combinación con antagonista 5 HT3 más dexametasona es recomendado por las guías MASCC con un nivel de confianza moderado, ESMO evidencia II y grado de recomendación B. Bajo potencial (fase aguda): dexametasona 12 mg (fase tardía): no precisa. Mínimamente emético. Solo será necesario el tratamiento en caso de aparición de NAVIQ tras el primer ciclo o con ciclos de potencial emético similar. Está indicado el uso de metoclopramida $10-40$ mg/6-8 horas o dexametasona 8-12 mg/24 horas[7],[12].

\section{Tratamiento de las náuseas y vómitos posoperatorios}

Actualmente, el tratamiento para la náusea y vomito en el postoperatorios en los pacientes oncológicos se mantiene subestimado e infratratado ya que las selecciones de los medicamentos adecuados se ven sesgados por la accesibilidad a los mismos o a una buena clasificación y estatificación de los pacientes por lo que se da una descripción de los medicamentos con lo que podemos utilizar en esta patología.

\section{Las fenotiazinas}

La metoclopramida es considerada un antiemético débil. Una dosis de 10 mg iv no es efectiva para reducir significativamente las NVPO1[13], es un fármaco gastrocinético, con propiedades dopaminérgicas, que resultan especialmente eficaces en la prevención de vómitos postoperatorios; es más útil en combinación con otros antieméticos, bloqueando los receptores dopaminérgicos, especialmente los de tipo D2 en el área de excitación de los quimiorreceptores, sin presentar actividad antipsicótica o tranquilizante.

Igualmente, es menos sedante que otros antagonistas de la dopamina, que a dosis bajas tiene un efecto antagonista dopaminérgico y a dosis altas ejerce un efecto antiserotoninérgico (anti HT-3). Su utilidad está restringida por reacciones extrapiramidales y según la dosis puede producir hipercinesia. Las recomendaciones más relevantes se dividen en: el paciente con categoría de riesgo baja o mínima de emesis (según guías MASCC 2016)[9].

Además, se recomienda en pacientes con náuseas y vómitos por cáncer avanzado. La dosis máxima recomendada es de 30 mg/día. Recomendación baja: Para el tratamiento de NVIQ altamente emetogénica. No recomendado en procesos de obstrucción intestinal parcial, terapia o en otra combinación fuera de la previamente descrita. Es un tratamiento alternativo en pacientes con náuseas y vómitos inducidos por cáncer avanzado[13],[11].

\section{Antagonistas del receptor de 5-hidroxitriptamina}

Es un tipo de antiemético, también llamado antagonista del receptor de 5-hidroxitriptamina 3 o antagonista del receptor de serotonina tipo 3.

\section{Ondansetrón}

Es el estándar de oro para la prevención de las NVPO, teniendo un mayor efecto en la prevención de vómitos que náuseas. Tiene una dosis recomendada de $4 \mathrm{mg}$ iv y $8 \mathrm{mg}$ vía oral (vo) Es tan efectivo como otros antagonistas del receptor de 5-hidroxitriptamina (5-HT3), incluyendo granisetrón 1-3 mg, así como la dexametasona $4 \mathrm{mg}$ y haloperidol $1 \mathrm{mg}$ iv. Los antagonistas 5-HT3 son más efectivos en la profilaxis de NVPO cuando son administrados al final del procedimiento quirúrgico, aunque el palonosetrón se indica típicamente al comienzo de la cirugía[14].

Los antagonistas 5-HT3 presentan buen perfil de seguridad, sin embargo, el palonosetrón prolongan el intervalo QT del electrocardiograma en dosis superiores a $16 \mathrm{mg}$ iv. Otros efectos adversos descritos se encuentran la cefalea, elevación de enzimas hepáticas y estreñimiento, aunque poco frecuentes[14].

\section{Palonosetrón}

Es el antagonista de serotonina más nuevo y más selectivo, con una afinidad 100 veces mayor al receptor de la serotonina, es uno de los tratamientos más efectivos para las náuseas y los vómitos inducidos por la quimioterapia. La alta afinidad parece no ser la única razón de la alta relación efectiva y hay signos de reestructuración del receptor que juegan un importante papel también[14].

Con una vida media prolongada de 40 horas, el palonosetrón proporciona efectos terapéuticos que duran por un período de 72 horas. La dosis habitual para inducir quimioterapia las náuseas y los vómitos son de 0,25 mg y de NVPO 0,075 mg[15]. Después de una sola dosis IV, más del $80 \%$ de una dosis única de la medicación es la excretado en la orina durante los próximos 6 días y la mitad de la cantidad no se modifica. No es necesario ajustar la dosis en pacientes geriátricos o con insuficiencia renal o discapacidad hepática. La seguridad y la eficacia no se han establecido en niños menores de 18 años de edad[14]. 


\section{Antagonistas de los NK-1}

Los receptores NK-1 tienen un papel muy importante en la emesis retardada. Existen inhibidores altamente selectivos de los receptores NK-1, de los que destacan el aprepitant y el fosaprepitant, los cuales son de gran utilidad en las NVPO refractarios al tratamiento y que se presentan después de las 12 horas de la cirugía[28]. Los antagonistas del receptor de NK1 mejoran el control de las náuseas y vómitos inducidos por quimioterapia en las fases aguda y tardía, en general, en los pacientes que recibieron quimioterapia alta y moderadamente emetogénica como se describió anteriormente. Ambos fármacos están indicados en el control de náuseas y vómitos inducidos por quimioterapia alta y moderadamente emetogénica en las fases aguda y tardía desde el primer ciclo y subsecuentes. Están indicados, además, en la prevención de NVPO a dosis de $40 \mathrm{mg} 3$ horas antes de iniciar el procedimiento anestésico[16].

Berrío Valencia, en su revisión sistemática de 2014, demuestra que el aprepitant se puede utilizar para la profilaxis del vómito a las 24 y 48 horas del postoperatorio en pacientes de alto riesgo, pero hacen falta más estudios para establecer su dosis efectiva 95 para la prevención de la náusea y vómito postoperatorio, así como en las poblaciones especiales como lo sería la población pediátrica[15],[16].

\section{Corticoides}

La dexametasona puede ser administrada como profilaxis o tratamiento para las NVPO. Una dosis profiláctica de 4-5 mg iv es recomendada después de la inducción anestésica en pacientes con riesgo de presentar esta complicación[14].

Su eficacia en la profilaxis es similar a la del ondansetrón $4 \mathrm{mg}$ iv y droperidol 1,25 mg iv. Estudios recientes prefieren una dosis de $8 \mathrm{mg}$ iv, en vez de la dosis mínima de 4-5 mg ya que esta dosis mejora la calidad de la recuperación postoperatoria global, además de reducir significativamente las náuseas, el dolor y la fatiga[11],[17]

Por otro lado, tiene un efecto analgésico leve a moderado, disminuyendo el uso de opioides postoperatorios. Al recibir $8 \mathrm{mg}$ iv, la dexametasona sí produce aumento significativo de la glucemia intra y postoperatoria inmediata, en pacientes con intolerancia a la glucosa, diabéticos tipo 2 y sometidos a cirugía bariátrica. Recomendamos utilizar solo $4 \mathrm{mg}$ iv del fármaco en este tipo de pacientes para evitar una hiperglucemia postoperatoria significativa[11],[17].

Como punto de referencia y de selección de pacientes obesos en la publicación de Aurelien Bataille en el 2016, llegan a la conclusión que la combinación de dexametasona y ondansetrón no fue efectiva en la prevención de NVPO o NVPO graves en pacientes obesos sometidos a gastrectomía en manga laparoscópica después de TIVA. La metilprednisolona 40 mg iv también es efectiva como profilaxis, con efectos adversos similares a la dexametasona, pero con mayor costo[18].

\section{Olanzapina}

Es un antipsicótico de la clase de las tienobenzodiazepinas, inhibe la liberación y/o unión de múltiples neurotransmisores con sus receptores específicos: dopamina en D1, D2, receptores cerebrales D3 y D4, serotonina en receptores 5-HT2a, 5-HT2c, 5-HT3 y 5-HT6, catecolaminas en receptores alfa adrenérgicos, acetilcolina en los receptores muscarínicos e histamina en los receptores H1[19],[20].

Tiene una gran afinidad por el receptor $5 \mathrm{HT} 2 \mathrm{a}$, que es hasta 5 veces mayor que el receptor de dopamina, lo que resulta en una menor propensión al desarrollo de efectos secundarios extrapiramidales. Entre sus efectos adversos se incluyen somnolencia, hipotensión postural, estreñimiento, mareos, inquietud y aumento de peso.

Smith et al., en su artículo de revisión comentan que la olanzapina disminuyó las náuseas y los vómitos inducidos por morfina. A una dosis que tenía un efecto antiemético $(0,03 \mathrm{mg} / \mathrm{kg})$ no indujo catalepsia o hiperglucemia, y no tuvo ningún efecto sobre la inhibición del tránsito gastrointestinal. La olanzapina también inhibe la hiperalgesia térmica y alivió por completo las alteraciones del sueño[21].

En su estudio comparativo con ondansetrón desarrollado por Chelkeba concluyen que la olanzapina se puede usar de forma efectiva para profilaxis contra NVPO, fue más efectiva en el período posoperatorio tardío y la dosis de olanzapina $10 \mathrm{mg}$ fue comparable a la dosis de ondansetrón de $16 \mathrm{mg}$ descrita en la literatura como "la dosis única oral óptima", también que se debe utilizar con precaución en los pacientes con enfermedades cardíacas, debido a que puede producir hipotensión ortostática y la hipotensión producida por hipovolemia, deshidratación o presencia de fármacos antihipertensivos[22]. En asociación con aprepitant o fosaprepitant más dexametasona en fase aguda y tardía en pacientes con mal control de NVIQ, posterior a primer ciclo con aprepitant o fosaprepitant más dexametasona, su uso también se indica en pacientes que reciben dosis altas de quimioterapia, como monoterapia o en otra combinación fuera de la previamente descrita.

Se considera actualmente que es un tratamiento alternativo en pacientes con náuseas y vómitos inducidos por cáncer avanzado; otras opciones son halo- 
peridol o levomepromazina. Y de suma importancia se debe considerar su efecto de sedación, sobre todo en población anciana[23].

\section{Cannabinoides THC (Delta-9-tetrahidrocannabinol)}

Los efectos estudiados del cannabis en el organismo son mediados por la unión de cannabinoides exógenos a receptores de endocannabinoides (CB1 y CB2) ampliamente distribuidos en el organismo. Se han descrito 66 cannabinoides exógenos, de los cuales el Delta-9-tetrahidrocannabinol (D9-THC) constituye el componente psicoactivo a través de su unión al receptor CB1, produciendo un efecto de agonista parcial, por lo que su efecto es dosis dependiente, es decir, a dosis bajas funciona como antiemético y a dosis altas hiperémesis. Se sabe que existe un antagonista de estos receptores Ilamado rimonabant[23].

Se ha descrito efectos adversos asociados al uso medicinal de los cannabinoides a través de diferentes vías (oral, sublingual, tópica, fumada, mezclada con alimentos o mediante infusiones), incluyen mareos, sequedad de boca, náuseas, fatiga, somnolencia, euforia, vómitos, desorientación, confusión, pérdida de equilibrio y alucinación, por los cuales existen ciertas restricciones para el uso de estos fármacos tales como adicción, sin embargo, está descrita la presencia de esta sólo en $10 \%$ de los consumidores de cannabinoides de manera recreativa.

Los cannabinoides han demostrado ser efectivos en la fase tardía de la náusea asociada a quimioterapia (24 horas posteriores a la administración), a diferencia de los antagonistas de receptores serotoninérgicos 5-HT3, los cuales tienen pobre respuesta en estos casos; probablemente la fisiopatología en los cuados de emesis aguda y tardía sea distinta. Los cannabinoides pueden bloquear los receptores 5-HT3[11],[23], además de esto se ha descrito también un síndrome de hiperémesis asociado al uso de cannabinoides, sin embargo, no existen estudios concluyentes, pues los factores de riesgo para desarrollar este síndrome son parecidos a los factores para desarrollar náusea y vómito posoperatorio; no existe un tiempo específico en el cual los pacientes hayan presentado este síndrome posterior al inicio del uso de cannabinoides así como el tratamiento es muy diverso y no sigue una línea para pensar que los cannabinoides es la razón de la hiperémesis en estos pacientes.

El uso de nabilona (vía oral) tiene variados efectos en el sistema nervioso central, por lo que muchos pacientes tienden a descontinuarlo al poco tiempo de iniciar su uso. Personas que han recibido la nabilona en forma de Cesamet $^{\circledR}$ (USA) han experimentado un incremento de la frecuencia cardíaca, cambios en el estado de ánimo (euforia, depresión, ansiedad, pánico), delusiones, alteración de la conducta y alucinaciones. Este efecto está asociado al uso de dosis elevadas de nabilona, aun así, se reporta casos de psicosis en pacientes que recibieron dosis bajas dentro del rango terapéutico[23].

El dronabinol, un derivado sintético de la delta 9-tetrahidrocanabinol que en Estados Unidos se comercia como Marinol ${ }^{\circledR}$ (tabletas) y Syndros ${ }^{\circledR}$ (dronabinol líquido), está indicado en la estimulación del apetito de pacientes oncológicos y con $\mathrm{VIH}$. A pesar de ello, se ha reportado actividad epiléptica en pacientes que recibieron dronabinol. Además, puede resultar adictivo y debe ser usado con precaución en personas con antecedentes de abuso de sustancias, manía, depresión o esquizofrenia, puesto que las cápsulas de dronabinol podrían exacerbar estos trastornos. En adultos mayores se debe monitorizar su titulación por su sensibilidad a posibles efectos hipotensores, psicoactivos y neurológicas del fármaco[23].

La revisión de la literatura muestra que los cannabinoides son más eficaces que el placebo y comparables a antieméticos como proclorperazina (fenoriazina) y ondansetrón. Las muestras de los estudios, los diseños y los resultados de seguridad son bastante heterogéneos. No ha sido recomendado aún por ninguna guía internacional. Octreotida, haloperidol, anticolinérgicos antisecretores como escopolamina, butilbromida, así como algunos esteroides, son altamente recomendados en las náuseas y vómitos secundarios a obstrucción intestinal[12],[11],[23].

\section{Butirofenonas}

\section{Droperidol}

Derivado de la butirofenona similar al haloperidol, antagonista de la dopamina que tiene propiedades ansiolíticas, sedantes, hipnóticas y antieméticas, su dosis profiláctica está entre 0,625 y 1,25 mg/iv, siendo efectiva para prevenir NVPO, su inicio de la acción farmacológica de droperidol ocurre dentro de 3 a 10 minutos, pero los efectos farmacológicos máximos pueden no ser aparentes hasta 30 minutos, similar al ondansetrón y la dexametasona[14].

Es más eficaz cuando se administra al final del procedimiento quirúrgico. En un gran número de estudios han documentado un efecto similar en la prolongación del intervalo QT y arritmias cardíacas graves, incluyendo Torsades de Pointes, se recomienda que 
se debe obtener un electrocardiograma de 12 derivaciones de todos los pacientes antes la administración de droperidol, y el medicamento no debe administrarse en hombres o pacientes mujeres con un intervalo QTc superior a 440 o 450 mseg, respectivamente, la monitorización debe continuarse durante 2 a 3 horas después de la administración del medicamento[15]. Este medicamento, por lo descrito anteriormente debe usarse con extrema precaución en los pacientes que con riesgo de desarrollo de síndrome de QT prolongado ( $p$. ej., insuficiencia cardíaca congestiva, bradicardia, uso de un diurético, hipertrofia cardíaca, hipocalemia, hipomagnesemia y uso de medicamentos conocidos por prolongar el intervalo QT). El utilizar ambos fármacos combinados es más efectivo sobre la prevención de NVPO que administrar cada fármaco por separado, no así la prolongación del intervalo QT, en donde la combinación de ambos fármacos no potencia este efecto[14].

\section{Haloperidol}

A dosis bajas (0,5-2 mg iv o im) está recomendado como antiemético, con una NNT de 4 y 6 para náuseas y vómitos, respectivamente. Su efectividad es comparable a la de droperidol u ondansetrón, tiene una vida media más larga que el droperidol, pero muestra menos especificidad por el receptor D2[24],[25].

Su uso también está asociado a una prolongación del intervalo QT. La incidencia de reacciones adversas a los neurolépticos es proporcional a la dosis. El droperidol es tan bien tolerado como los setrones, aunque con más sedación (de manera dependiente de dosis). Con el uso de los neurolépticos a dosis más altas que las recomendadas pueden aparecer ansiedad, inquietud, síntomas extrapiramidales e incluso síndrome neuroléptico maligno.

También tienen propiedades antieméticas al utilizarse en dosis más bajas que las usadas para tratar enfermedades psiquiátricas y se ha investigado como una alternativa aldroperidol. Dosis de 0,5-2 mg intramuscular o iv son efectivas en reducir el riesgo de NVPO, de forma similar al droperidol, no ocurriendo sedación ni arritmias cardiacas significativas. Sin embargo, conlleva un riesgo mayor de prolongación del intervalo QT del electrocardiograma, no siendo recomendable como primera línea profiláctica o terapéutica[26].

\section{Benzodiazepinas}

\section{Midazolam}

En el año 2016, Grant y colaboradores, publica- ron un metaanálisis de 12 estudios, el cual concluye que la administración de midazolam iv, entre 2 y 5 mg, en el período pre o intraoperatorio, reducía significativamente las NVPO, náuseas o vómitos por sí solos, y el uso de antieméticos de rescate durante las primeras $24 \mathrm{~h}$ postoperatorias. Se observa una diferencia significativa en la aparición de efectos adversos de midazolam, como sedación y depresión respiratoria. Así mismo Ahn y colaboradores en el mismo año (2016), en su revisión sistemática y metaanálisis de 16 estudios, en los cuales se administró midazolam profiláctico en el pre o postoperatorio, se encontro una incidencia significativamente menor de náuseas exclusivas, vómitos exclusivos y NVPO, comparado con placebo[27].

Lee et al., en publicaciones anteriores publicadas en 2007 con un total de 30 publicaciones, refieren que al administrar $2 \mathrm{mg}$ iv del fármaco 30 min antes de la cirugía resultó igual de efectivo en la prevención de NVPO que $4 \mathrm{mg}$ de ondansetrón. El administrar midazolam con dexametasona resultó más efectivo en la profilaxis de NVPO que utilizar cada uno por separado, demostrando un efecto sinérgico[28].

\section{Lorazepam}

Benzodiazepina con efecto ansiolítico y actividad hipnótica, anticonvulsivante, sedante, relajante muscular y amnésica. Su efecto antiemético está relacionado con la combinación de efectos de sedación, reducción de la ansiedad y posible depresión en el centro activador del vómito, indicado como coadyuvante con fármacos antieméticos para el tratamiento de náuseas y vómitos asociados con la quimioterapia, porque evita los síntomas de las náuseas anticipatorias y las náuseas y vómitos tardíos, con dosis: 0,5-2 mg vo/iv cada 6 horas y en ancianos: 0,5 mg/ día[14].

\section{Propofol}

Numerosos estudios han demostrado sus propiedades antieméticas, Utilizado como parte de (TIVA) anestesia total intravenosa es altamente recomendado para reducir el riesgo basal de (NVPO), disminuyendo su incidencia hasta en $25 \%$. Para la inducción y mantención de la anestesia disminuye significativamente la incidencia de (NVPO) dentro de las primeras 6 h postoperatorias. Una revisión sistemática demostró que utilizar propofol iv en comparación con usar anestesia inhalatoria también disminuye significativamente el riesgo de (NVPO) $C$.

Este fármaco se puede utilizar como terapia de rescate en caso de (NVPO) persistentes en la Unidad de Recuperación, usando pequeñas dosis de entre 
10 y $20 \mathrm{mg}$ iv, siendo tan efectivo como el ondansetrón[29].

Naghibi y colaboradores, en su estudio publicado en 2015, utilizaron diferentes dosis de propofol 20 $\mathrm{mg}$, propofol $30 \mathrm{mg}$ y metoclopramida $10 \mathrm{mg}$ demostrando que la incidencia de NVPO fue menor durante las primeras $6 \mathrm{~h}$ con dosis de propofol $20 \mathrm{mg}$. Sin embargo, tiene el inconveniente de tener un efecto antiemético breve y complicaciones asociadas, como hipotensión arterial, apnea/hipopnea y sedación[30].

\section{OTROS MEDICAMENTOS}

\section{Los antihistamínicos}

Actualmente, son un fármaco de segunda o tercera línea para la profilaxis y tratamiento de (NVPO). La escopolamina transdérmica es un anticolinérgico que sería útil para prevenir las (NVPO) como coadyuvante de otras terapias antieméticas, administrada el día previo a la cirugía o $2 \mathrm{~h}$ previas al inicio de la anestesia[31].

\section{Los Alfa-2 agonistas}

En la publicación Blaudszun et al., refieren que tanto la clonidina y dexmedetomidina, mostraron solo en un metaanálisis un significativo efecto contra la aparición de náuseas postoperatorias, no así contra los vómitos[32].

\section{Mirtazapina}

Antidepresivo noradrenérgico y serotoninérgico, útil como un coadyuvante de otras terapias antieméticas, como $30 \mathrm{mg}$ vo asociado a dexametasona $8 \mathrm{mg}$ iv[14].

\section{Meclizina}

Administrado en dosis de $50 \mathrm{mg}$ vo y asociado a ondansetrón $4 \mathrm{mg}$ iv sería más efectivo en la profilaxis de (NVPO) que utilizar cualquiera de los 2 medicamentos por sí solo[14].

\section{Gabapentinoides}

Gabapentina en dosis de 600 y 800 mg vo, administrada $1 \mathrm{~h}$ previa a la cirugía, disminuiría eficazmente las (NVPO), similar a dexametasona. La combi- nación de gabapentina y dexametasona es mejor que administrar cada una por sí sola[14],[33].

\section{Algoritmo de manejo médico}

De acuerdo a las actualizaciones presentadas por Roila F, Molassiotis A, Paul J. Hesketh de las guías medicas de NVPO la Multinational Association of Supportive Care in Cancer/European Society for Medical Oncology (MASCC/ESMO), la Sociedad Americana de Oncología Clínica (ASCO) y la National Comprehensive Cancer Network (NCCN). Se anexan las dosis y medicamentos indicados según su riesgo Emético y se anexa en Tabla 2[7],[2].

Altamente eméticos: Antagonista receptor 5HT3 + más antagonista NK1 + dexametasona (en caso de ser refractario \pm olanzapina). Moderadamente eméticos: Antagonista 5HT3 + dexametasona $12 \mathrm{mg}$. Días 2-4 (fase tardía): dexametasona 8 $\mathrm{mg} \pm$ antagonistas 5HT3. Moderadamente eméticos basados en carboplatino: NK1 + Antagonista 5HT3 + dexametasona $12 \mathrm{mg}$. Moderadamente eméticos (sin carboplatino): Antagonista 5HT3 + dexametasona $12 \mathrm{mg}$. Mínimamente emético: Solo será necesario el tratamiento en caso de aparición de NAVIQ tras el primer ciclo o con ciclos de potencial emético similar.

\section{Conclusiones}

Las NVPO no constituyen un problema vital, pero son frecuentes y de difícil control, especialmente en pacientes de alto riesgo, por lo que es importante conocer los algoritmos actuales de manejo de (NVPO) y los antieméticos de nueva generación disponibles. Mientras tanto, debemos saber hasta qué punto se conocen y se aplican las guías clínicas y qué incidencia de NVPO en nuestros hospitales.

En este entorno es primordial que el anestesiólogo recuerde que cada paciente es único, diferente y evalúe de forma integral el riesgo de (NVPO) dentro de la valoración preoperatoria, para establecer el tratamiento adecuado administrando una profilaxis en el número, dosis y tiempo correctos de la gama de medicamentos antieméticos con lo que se tienen actualmente. 


\begin{tabular}{|c|c|c|}
\hline \multicolumn{3}{|c|}{ Tabla 2} \\
\hline Clasificación & Medicamentos & Dosis \\
\hline $\begin{array}{l}\text { Pacientes con alto riesgo } \\
(90 \%)\end{array}$ & 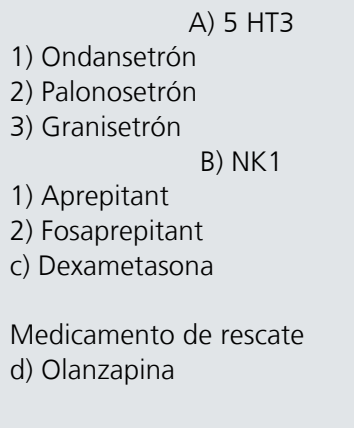 & 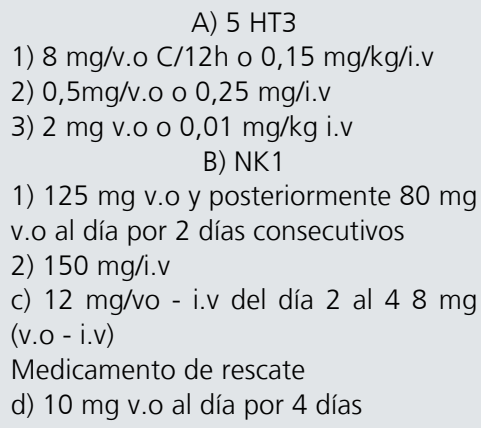 \\
\hline $\begin{array}{l}\text { Pacientes con moderado riesgo } \\
(30-89 \%)\end{array}$ & $\begin{array}{l}\text { A) } 5 \text { HT3 } \\
\text { 1) Ondansetrón } \\
\text { 2) Palonosetrón } \\
\text { 3) Granisetrón } \\
\text { B) Dexametasona }\end{array}$ & $\begin{array}{l}\text { A) } 5 \text { HT3 } \\
\text { 1) } 8 \mathrm{mg} / \mathrm{v} . \circ \mathrm{C} / 12 \mathrm{~h} \mathrm{o} 0,15 \mathrm{mg} / \mathrm{kg} / \mathrm{i} . \mathrm{v} \\
\text { 2) } 0,5 \mathrm{mg} / \mathrm{v} . \circ \circ 0,25 \mathrm{mg} / \mathrm{i} . \mathrm{v} \\
\text { 3) } 2 \mathrm{mg} \text { v.o o } 0,01 \mathrm{mg} / \mathrm{kg} \mathrm{i.v} \\
\text { b) } 12 \mathrm{mg} / \mathrm{v} .0-\text { - I.V del día } 2 \text { al } 48 \mathrm{mg} \\
\text { (v.o - I.V) }\end{array}$ \\
\hline $\begin{array}{l}\text { Pacientes con bajo riesgo } \\
(10-29 \%)\end{array}$ & $\begin{array}{l}\text { A) } 5 \text { HT3 } \\
\text { 1) Ondansetrón } \\
\text { 2) Palonosetrón } \\
\text { 3) Granisetrón } \\
\text { B) Dexametasona }\end{array}$ & $\begin{array}{l}\text { A) } 5 \mathrm{HT} 3 \\
\text { 1) } 8 \mathrm{mg} / \mathrm{v} . \circ \mathrm{C} / 12 \mathrm{~h} \circ 0,15 \mathrm{mg} / \mathrm{kg} / \mathrm{l} . \mathrm{V} \\
\text { 2) } 0,5 \mathrm{mg} / \mathrm{v} .0 \text { o } 0,25 \mathrm{mg} / \mathrm{i} . \mathrm{v} \\
\text { 3) } 2 \mathrm{mg} \mathrm{v} .0 \circ 0,01 \mathrm{mg} / \mathrm{kg} \mathrm{i.v} \\
\text { b) } 8 \mathrm{mg} / \mathrm{v} . \mathrm{O}-\mathrm{I} . \mathrm{V}\end{array}$ \\
\hline $\begin{array}{l}\text { Paciente con riesgo mínimo } \\
(-10 \%)\end{array}$ & $\begin{array}{l}\text { a) Metoclopramida } \\
\text { b) Dexametasona }\end{array}$ & $\begin{array}{l}\text { a) } 10-40 \mathrm{mg} \text { cada } 6-8 \mathrm{~h} \\
\text { b) } 8-12 \mathrm{mg} \mathrm{C} / 24 \mathrm{~h}\end{array}$ \\
\hline
\end{tabular}

v.o (vía oral) i.v (intra venoso).

\section{Referencias}

1. Nazar JC, Bastidas EJ, Coloma DR, Zamora H. M, Fuentes HR. Prevención y tratamiento de pacientes con náuseas y vómitos postoperatorios. Vol. 69. Rev Chil Cir. 2017;69(5):421-8. https://doi.org/10.1016/j. rchic.2017.02.009.

2. Carlos LA, Sergio QE. Profilaxis y tratamiento de las náuseas y vómitos postoperatorios. Rev Chil Anest. 2009;38(1):24-33.

3. Apfel CC, Heidrich FM, JukarRao S, Jalota L, Hornuss C, Whelan RP, et al. Evidence-based analysis of risk factors for postoperative nausea and vomiting. In: British Journal of Anaesthesia. 2012. p. 742-53. https://doi. org/10.1093/bja/aes276.

4. Gan TJ, Diemunsch P, Habib AS, Kovac A, Kranke P, Meyer TA, et al.; Society for Ambulatory Anesthesia. Consensus guidelines for the management of postoperative nausea and vomiting. Anesth Analg. 2014 Jan;118(1):85-113. https://doi.org/10.1213/ ANE.0000000000000002 PMID:24356162

5. Apfel CC, Philip BK, Cakmakkaya OS, Shilling A, Shi YY, Leslie JB, et al. Who is at risk for postdischarge nausea and vomiting after ambulatory surgery? Anesthesiology. 2012 Sep;117(3):475-86. https://doi.org/10.1097/ ALN.0b013e318267ef31

\section{PMID:22846680}

6. García Gómez J, Pérez López ME, Alonso Bermejo M, Escobar Álvarez Y, García Mata J; SEOM. SEOM guide to antiemetic prophylaxis in cancer patients treated with chemotherapy 2013. Clin Transl Oncol. 2013 Dec;15(12):1030-6. https://doi. org/10.1007/s12094-013-10932 PMID:24019035

7. Basch E, Hesketh PJ, Kris MG, Prestrud AA, Temin S, Lyman $\mathrm{GH}$. Antiemetics: american society of clinical oncology clinical practice guideline update. J Oncol Pract. 2011 Nov;7(6):395-8. https://doi. org/10.1200/JOP.2011.000397 PMID:22379425

8. Grunberg SM, Osoba D, Hes- 
keth PJ, Gralla RJ, Borjeson S, Rapoport BL, et al. Evaluation of new antiemetic agents and definition of antineoplastic agent emetogenicity-an update. Support Care Cancer. 2005 Feb;13(2):80-4. https:// doi.org/10.1007/s00520-0040718-y PMID:15599601

9. Roila F, Molassiotis A, Herrstedt J, Aapro M, Gralla RJ, Bruera $E$, et al.; participants of the MASCC/ESMO Consensus Conference Copenhagen 2015. 2016 MASCC and ESMO guideline update for the prevention of chemotherapy- and radiotherapy-induced nausea and vomiting and of nausea and vomiting in advanced cancer patients. Ann Oncol. 2016 Sep;27 suppl 5:v119-33. https://doi. org/10.1093/annonc/mdw270 PMID:27664248

10. Valle $A E$, Cervantes $G$, Franco EE, García G, Hernández GA, López M, et al. Guías de Manejo de Antieméticos en Oncología, Hematología y Radioterapia. Gac Mex Oncol. 2011;10 S4:3-11.

11. Cervantes G, Ochoa FJ, Acosta A, Alvarado M, Arreguín FE, Enríquez Ml, et al. Guía para el manejo de antieméticos en oncología, hematología y radioterapia. Gac Mex Oncol. 2016;15 S2:3-34.

12. Berger MJ, Ettinger DS, Aston J, Barbour S, Bergsbaken J, Bierman PJ, et al. NCCN Guidelines Insights: Antiemesis, Version 2.2017. J Natl Compr Canc Netw. 2017 Jul;15(7):883-93. https://doi. org/10.6004/jnccn.2017.0117 PMID:28687576

13. Valle $A E$, Cervantes $G$, Franco EE, García G, Hernández GA, López M, et al. Guías de Manejo de Antieméticos en Oncología, Hematología y Radioterapia. Gac Mex Oncol. 2011;10 S4:3-11.

14. Cervantes G, Ochoa FJ, Acosta
A, Alvarado M, Arreguín FE, Enríquez MI, et al. Guía para el manejo de antieméticos en oncología, hematología y radioterapia. Gac Mex Oncol. 2016;15 S2:3-34.

15. Kaye AD, Cornett EM, Chalabi J, Naim NZ, Novitch MB, Creel JB, et al. Pharmacology of Antiemetics: Update and Current Considerations in Anesthesia Practice. Anesthesiol Clin. 2017 Jun;35(2):e41-54. https://doi.org/10.1016/j. anclin.2017.01.003 PMID:28526160

16. Carrillo R, Espinoza I, Nava JA. Náusea y vómito postoperatorio. Rev Mex Anestesiol. 2012;35(2):122-31.

17. Bodge $M$, Shillingburg $A$, Paul S, Biondo L. Safety and efficacy of aprepitant for chemotherapyinduced nausea and vomiting in pediatric patients: a prospective, observational study. Pediatr Blood Cancer. 2014 Jun;61(6):1111-3. https:// doi.org/10.1002/pbc.24901 PMID:24357337

18. De Oliveira GS Jr, Almeida MD, Benzon HT, McCarthy RJ. Perioperative single dose systemic dexamethasone for postoperative pain: a metaanalysis of randomized controlled trials. Anesthesiology. 2011 Sep;115(3):575-88. https://doi.org/10.1097/ ALN.0b013e31822a24c2 PMID:21799397

19. Bataille $A$, Letourneulx JF, Charmeau $A$, Lemedioni $P$, Léger $\mathrm{P}$, Chazot $\mathrm{T}$, et al. Impact of a prophylactic combination of dexamethasone-ondansetron on postoperative nausea and vomiting in obese adult patients undergoing laparoscopic sleeve gastrectomy during closed-loop propofol-remifentanil anaesthesia: A randomised double-blind place. In: European Journal of
Anaesthesiology. 2016. p. 898905. https://doi.org/10.1097/ EJA.0000000000000427.

20. Mizukami N, Yamauchi M, Koike K, Watanabe A, Ichihara K, Masumori $\mathrm{N}$, et al. Olanzapine for the prevention of chemotherapy-induced nausea and vomiting in patients receiving highly or moderately emetogenic chemotherapy: a randomized, double-blind, placebo-controlled study. J Pain Symptom Manage. 2014 Mar;47(3):542-50. https://doi.org/10.1016/j. jpainsymman.2013.05.003 PMID:23856100

21. Hesketh PJ, Bohlke K, Lyman $\mathrm{GH}$, Basch E, Chesney M, ClarkSnow RA, et al.; American Society of Clinical Oncology. Antiemetics: american Society of Clinical Oncology focused guideline update. J Clin Oncol. 2016 Feb;34(4):381-6. https://doi. org/10.1200/JCO.2015.64.3635 PMID:26527784

22. Smith HS, Smith JM, Seidner P. Opioid-induced nausea and vomiting. Ann Palliat Med. 2012 Jul;1(2):121-9. PMID:25841472

23. Chelkeba L, Gidey K, Mamo A, Yohannes B, Matso T, Melaku T. Olanzapine for chemotherapyinduced nausea and vomiting: systematic review and metaanalysis. Pharm Pract (Granada). 2017 Jan-Mar;15(1):877. https://doi.org/10.18549/ PharmPract.2017.01.877 PMID:28503222

24. Rojas $S$, Velázquez $P$, Segura M. Cannabinoides: utilidad actual en la práctica clínica. Acta Med Grupo Ángeles. 2015;13(4):244-50.

25. Veiga-Gil L, López-Olaondo L, Pueyo J, Callejas R, Duque P, Carrascosa F. Dosis bajas de haloperidol en combinación con ondansetrón no son eficaces para la profilaxis de náuseas y vómitos postoperatorios en pa- 
cientes propicios a esta complicación. Cir Esp. 2015;93(2):1106. https://doi.org/10.1016/j. ciresp.2014.01.011 PMID:24629922

26. Chaparro C, Moreno D, Ramírez V, Fajardo A, González D, Sanín A, et al. Haloperidol como profilaxis para náuseas y vómito postoperatorios: revisión sistemática. Rev Colomb Anestesiol. 2013 Jan;41(1):34-43. https://doi.org/10.1016/j. rca.2012.07.010.

27. Grant MC, Kim J, Page AJ, Hobson D, Wick E, Wu CL. The effect of intravenous midazolam on postoperative nausea and vomiting: A meta-analysis. Anesth Analg. 2016 Mar;122(3):65663. https://doi.org/10.1213/ ANE.0000000000000941 PMID:26332858

28. Ahn EJ, Kang H, Choi GJ, Baek $\mathrm{CW}$, Jung $\mathrm{YH}$, Woo $\mathrm{YC}$. The Effectiveness of Midazolam for Preventing Postoperative Nausea and Vomiting: A Systematic Review and Meta-Analysis. Anesth
Analg. 2016 Mar;122(3):664-

76. https://doi.org/10.1213/

ANE.0000000000001062 PMID:26516802

29. Lee $Y$, Wang JJ, Yang YL, Chen A, Lai HY. Midazolam vs ondansetron for preventing postoperative nausea and vomiting: a randomised controlled trial. Anaesthesia. 2007 Jan;62(1):1822. https://doi.org/10.1111/ j.1365-2044.2006.04895.x PMID:17156222

30. Unlugenc $H$, Guler T, Gunes Y, Isik G. Comparative study of the antiemetic efficacy of ondansetron, propofol and midazolam in the early postoperative period. Eur J Anaesthesiol. 2004 Jan;21(1):60-5. https:// doi.org/10.1097/00003643200401000-00010 PMID:14768925

31. Naghibi K, Kashefi P, Azarnoush $\mathrm{H}$, Zabihi P. Prevention of postoperative nausea and vomiting with a subhypnotic dose of Propofol in patients undergoing lower abdominal surgery: A prospective, randomized, double-blind study. Adv Biomed Res. 2015 Feb;4(1):35. https://doi. org/10.4103/2277-9175.151239 PMID:25789261

32. Wallenborn J, Gelbrich G, Bulst D, Behrends K, Wallenborn H, Rohrbach A, et al. Prevention of postoperative nausea and vomiting by metoclopramide combined with dexamethasone: randomised double blind multicentre trial. BMJ. 2006 Aug;333(7563):324. https://doi.org/10.1136/ bmj.38903.419549.80 PMID:16861255

33. Blaudszun G, Lysakowski C, Elia N, Tramèr MR. Effect of perioperative systemic $\alpha 2$ agonists on postoperative morphine consumption and pain intensity: systematic review and meta-analysis of randomized controlled trials. Anesthesiology. 2012 Jun;116(6):1312-22. https://doi.org/10.1097/ ALN.0b013e31825681cb PMID:22546966 